\title{
Etodolac Attenuates Hippocampal Blood Flow Responses Induced by Tooth Pulp Stimulation in Rats
}

\author{
Makoto Hasegawa $^{1}$, Junichi Hada ${ }^{2}$, Tetsuya Abe ${ }^{1}$, Nao Ohtsu ${ }^{1}$, Kousuke Honda ${ }^{1}$, Masahiro Urade ${ }^{1}$ \\ ${ }^{1}$ Department of Dentistry and Oral Surgery, Hyogo College of Medicine, Nishinomiya, Japan; ${ }^{2}$ Hirakata General Hospital for Devel- \\ opmental Disorders, Hirakata, Japan. \\ E-mail: m-hase@hyo-med.ac.jp
}

Received June $16^{\text {th }}$, 2011; revised July 20 ${ }^{\text {th }}, 2011$; accepted July 30 2011.

\begin{abstract}
In this study, we investigated whether increases in hippocampal blood flow (HBF) by tooth pulp stimulation (TPS) are mediated via the activation of cyclooxygenase-2 (COX-2). We measured mean arterial blood pressure (MABP) and $H B F$ in urethane-anesthetized rats using laser Doppler flowmetry and examined the effects of etodolac, a specific COX2 inhibitor, on TPS-induced MABP and HBF responses. The MABP response was not attenuated by the intraperitoneal (i.p.) administration of etodolac $(10 \mathrm{mg} / \mathrm{kg})$; however, the HBF response was significantly attenuated by etodolac administration. These results suggest that COX-2 may be involved in the mechanisms regulating TPS-induced HBF responses.
\end{abstract}

Keywords: Cyclooxygenase-2 (COX-2), Etodolac, Fear Memory, Hippocampal Blood Flow, Mean Arterial Blood Pressure, Tooth Pulp Stimulation

\section{Introduction}

A positive relationship between anxiety and pain is a common experience in the dental clinic [1,2]. Okawa et al. [2] discussed the effects of level of anxiety about dental treatment on pain during treatment. Regarding their discussion, Ploghous et al. [1] showed that anxietyinduced hyperalgesia is associated with activation of entorhinal cortex of the hippocampal formation and suggested that accurate dental preparation alleviated pain by disenganging the hippocampal formation. The hippocampal formation responded differentially to noxious stimuli, dependent on whether the received pain intensity was enhanced by pain-relevant anxiety; however, there are few reports regarding the relationship between tooth pulp stimulation (TPS) and hippocampal activity [3]. We previously showed that TPS increases hippocampal blood flow (HBF) in rats, and that the nonselective adenosine receptor antagonist, theophylline, attenuates TPS-induced HBF responses [4]. We suggested that TPS-induced HBF responses may be, at least in part, mediated through adenosine receptors. In that study, because the increases in TPS-induced HBF responses were not completely abolished by theophylline, other factors including extracellular potassium ions [5], nitric oxide [6], and cyclooxygenase (COX) reaction products [7] might have contributed. Therefore, in the present study, we hypothesized that some TPS-induced HBF responses may be mediated via the activation of COX-2. We measured HBF and mean arterial blood pressure (MABP) in rats and examined the effects of a selective COX-2 inhibitor, etodolac, on TPSinduced HBF responses.

\section{Materials and Methods}

\subsection{Animal Preparation}

All procedures were conducted with the approval of "The Animal Care and Use Committee of Hyogo College of Medicine" and according to "The Guiding Principles for Care and Use of Animals Approved by The Council of The Physiological Society of Japan”. The experimental procedures were essentially the same as described previously [4].

Male Wistar rats weighing between 320 - $380 \mathrm{~g}$ were used. The rats were anesthetized with urethane $(1.2 \mathrm{~g} / \mathrm{kg})$ and their rectal temperature was maintained at $37^{\circ} \mathrm{C}$ $38^{\circ} \mathrm{C}$ with a heating pad. The tips of both lower incisors were cut off with a dental bar and stainless steel wire 
electrodes were inserted into the pulp and fixed with acrylic resin. The rats were then placed into stereotaxic frames. MABP was measured by means of a pressure transducer through a canula inserted into the right common carotid artery. To measure HBF, a laser Doppler flowmetry probe (Needle Type ON97-066, tip diameter: $0.5 \mathrm{~mm}$; Unique Medical, Tokyo, Japan) was stereotaxically inserted into the left dorsal hippocampus $(5.5 \mathrm{~mm}$ caudal to the bregma, $4.0 \mathrm{~mm}$ lateral to the midline, and $3.0 \mathrm{~mm}$ below the cortical surface).

\subsection{Stimulation of the Pulp}

As TPS, the pulp of both lower incisors was stimulated electrically with a negative square wave using a constant isolated current source (isolator: SS-202J with an electronic stimulator: SEN-3301; Nihon Kohden, Tokyo, Japan). The threshold stimulus intensity was determined in each rat with a $0.5-\mathrm{ms}$ duration, at $100 \mathrm{~Hz}$, for $10 \mathrm{~s}$ by slowly increasing the current from zero until a clear HBF increase was observed. The mean threshold was $0.6 \mathrm{~mA}$. We used 5 times the threshold (5T) as the stimulus intensity and compared the TPS-induced maximum MABP and $\mathrm{HBF}$ response values before and at 30 and $60 \mathrm{~min}$ after methylcellulose or etodolac administration.

\subsection{Experimental Groups}

Six rats received methylcellulose solution as a control. To examine the involvement of COX-2 in TPS-induced $\mathrm{HBF}$ and MABP responses, another 12 rats received etodolac, a specific COX-2 inhibitor. These agents ware administrated intraperitoneally. The dose $(10 \mathrm{mg} / \mathrm{kg})$ of etodolac was based on a previous study [8]. The volume ( $5 \mathrm{ml} / \mathrm{kg}$ ) of administrated methylcellulose was the same as etodolac.

\subsection{Drugs}

Etodolac was provided by Nippon Shinyaku Corporation (Kyoto, Japan) and suspended in a $0.5 \%$ methylcellulose solution.

\subsection{Data Analysis}

All data are expressed as the means \pm standard error of the means (S.E.M.). To determine the differences between experimental groups regarding time courses of changes in MABP and HBF responses induced by TPS, statistical analysis was performed by mixed-type analysis of variance (ANOVA) with repeated measures. Differences were considered significant at $p<0.05$.

\section{Results}

Figure 1 shows the typical temporal patterns of TPSinduced HBF and MABP responses. The peak latency of maximum amplitude in TPS-induced HBF responses always was shorter than that in TPS-induced MABP responses in each experiment. TPS at 5T increased both MABP and HBF before $0.5 \%$ methylcellulose $(5 \mathrm{ml} / \mathrm{kg}$, i.p.) or etodolac (10 $\mathrm{mg} / \mathrm{kg}$, i.p.). ANOVA computed according to changes in MABP responses induced by TPS revealed that the main effects of group and time and the interaction between group and time were not significant $(\mathrm{F}(1 / 17)=1.314, p=0.268 ; \mathrm{F}(1 / 17)=0.053, p=0.949$; $\mathrm{F}(1 / 17)=1.287, p=0.290$, respectively) (Figure 2). The mean value of TPS-induced HBF responses measured before methylcellulose or etodolac administration was $7.9 \pm 0.9$ laser Doppler unit $(n=18)$. Because the measured HBF values were relative ones, HBF data shown in Figure 3 are expressed as a percentage of the value of

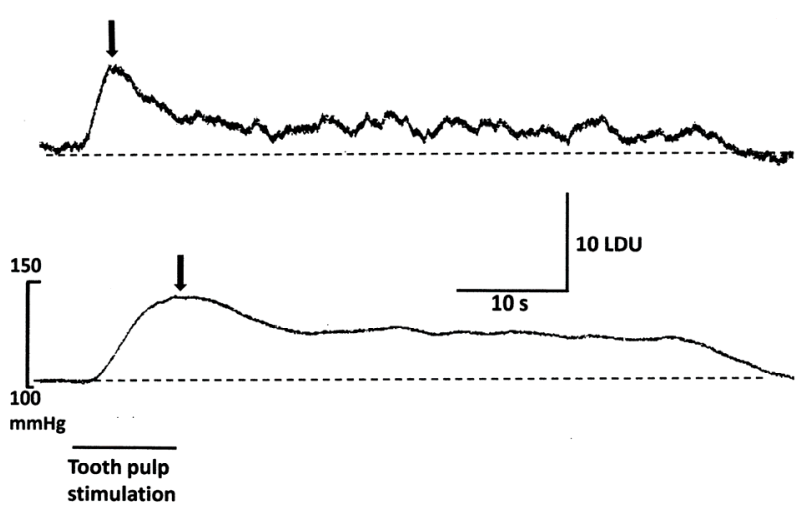

Figure 1. Representative records of TPS-induced HBF (upper trace) and MABP (lower trace) responses. The horizontal bar and downward arrows indicate the stimulation period (10 s) and the peak values of TPS-induced HBF and MABP responses, respectively. LDU, laser Doppler unit.

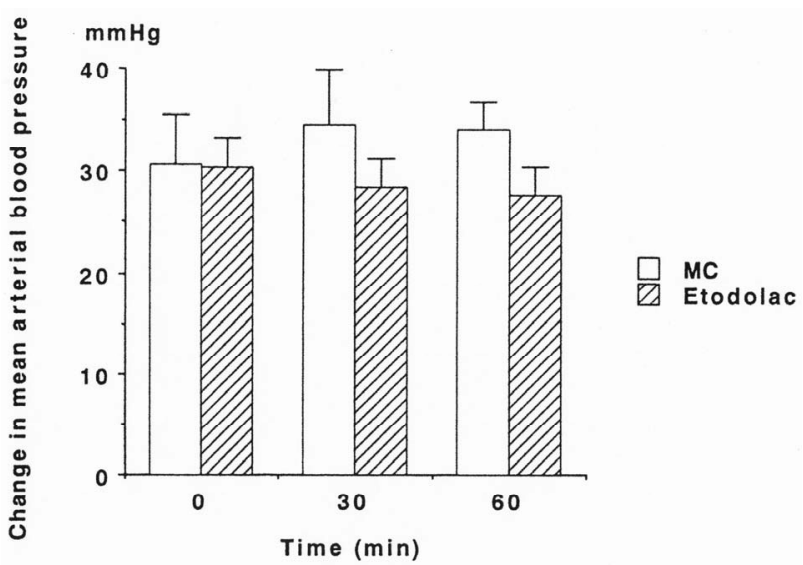

Figure 2. Effect of etodolac on TPS-induced MABP responses at 5 times the threshold (5T) before and at 30 and $60 \mathrm{~min}$ after methylcellulose (MC, $5 \mathrm{ml} / \mathrm{kg}$, i.p., open column) or etodolac $(10 \mathrm{mg} / \mathrm{kg}$, i.p., hatched column) administration. Values are the mean \pm S.E.M. (methylcellulose, $n=$ 6 ; etodolac, $n=12)$. These responses were not affected by etodolac. 


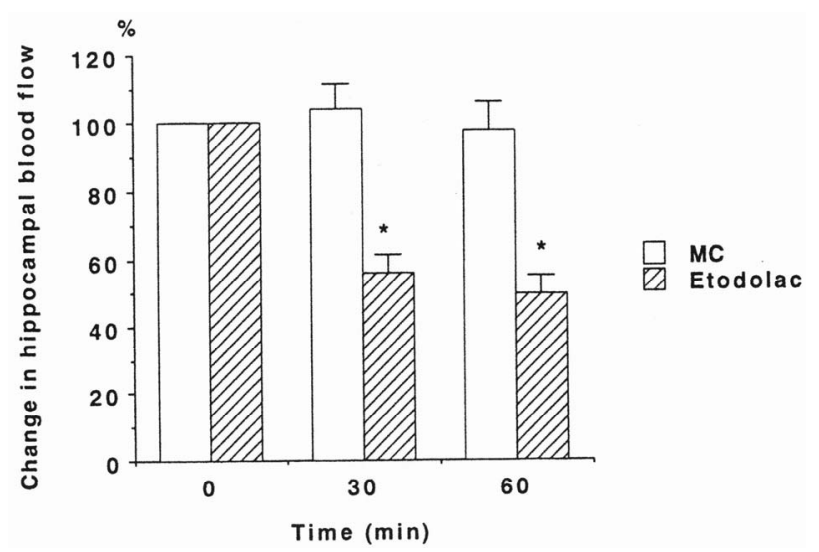

Figure 3. Effect of etodolac on TPS-induced HBF responses at 5 times the threshold (5T) before and at 30 and $60 \mathrm{~min}$ after methylcellulose (MC, $5 \mathrm{ml} / \mathrm{kg}$, i.p., open column) or etodolac $(10 \mathrm{mg} / \mathrm{kg}$, i.p., hatched column) administration. Values are the mean \pm S.E.M. (methylcellulose, $n=6$; etodolac, $n=12)$. The increases in HBF produced by TPS were significantly attenuated by etodolac administration $(p<$ 0.0001 vs MC).

each TPS-induced HBF response before methylcellulose or etodolac administration. Methylcellulose did not affect TPS-induced HBF responses at 30 and 60 min after administration. ANOVA computed according to changes in HBF responses induced by TPS revealed that the main effect of group was significant $(\mathrm{F}(1 / 17)=34.918, p<$ 0.0001 ), although the main effect of time and the interaction between group and time were not significant $(\mathrm{F}(1 / 17)$ $=2.529, p=0.131 ; \mathrm{F}(1 / 17)=0.002, p=0.962$, respectively) (Figure 3). Thus, etodolac significantly attenuated TPS-induced HBF responses at 30 and 60 min after administration.

\section{Discussion}

In the present study, we examined whether the inhibition of COX-2 modifies TPS-induced MABP and HBF responses. A specific COX-2 inhibitor, etodolac, attenuated TPS-induced HBF responses, but not MABP responses. To our knowledge, this is the first study in which etodolac attenuated TPS-induced HBF responses in rats.

The present data show that inhibition of COX-2 by etodolac may be one of the causal factors involved in the reduction of TPS-induced HBF responses. With regard to cerebral blood flow, similar data were previously obtained by Niwa et al. [9], who reported that a COX-2 inhibitor, NS-398, attenuates increases in somatosensory cortex flow produced by vibrissal stimulation. Thus, our data provide evidence that COX-2 is involved in the mechanisms regulating cerebral blood flow during synaptic activity.

In some organs, COX-2 is not present in a normal state, but its expression is induced by inflammatory stimulation [10]; however, COX-2 immunoreactivity is expressed and localized in a subpopulation of excitatory neurons in the neo- and allocortices, the hippocampus, and the amygdala [11]. Moreover, COX-2 immunoreactivity is present in dendritic spines.

Stimulation of the central nucleus of the amygdala, a limbic forebrain structure, produces changes in blood pressure and heart rate similar to those produced by stressful events [12]. Seidenbecher et al. [13] observed that Pavlovian fear conditioning induces an increase in the synchronization of theta-frequency activity in the lateral amygdala and CA1 region of the hippocampus. These findings suggest that activation of an amygdalahippocampus circuit is involved in fear-based learning. Thus, TPS-induced HBF responses may be involved in fear memory and anxiety related to tooth pain.

\section{Conclusions}

The findings of present study showed that pharmacological inhibition of COX-2 with etodolac attenuates TPSinduced HBF responses; however, TPS-induced MABP responses are not attenuated by etodolac. Our findings provide evidence of the involvement of COX-2 in TPSinduced $\mathrm{HBF}$ responses.

\section{Acknowledgements}

This work was partly supported by a Grant-in-Aid for Scientific Research (C) JSPS KAKENHI (21592438). We thank Professor Hiroyuki Iso, Department of General Education Center, Hyogo University of Health Sciences, for statistical analysis.

\section{REFERENCES}

[1] A. Ploghaus, C. Narain, C. F. Beckmann, S. Clare, S. Bantick, R. Wise, P. M. Matthews, J. N. P. Rawlins and I. Tracey, "Extracerbation of Pain by Anxiety is Associated with Activity in a Hippocampal Network," Journal of Neuroscience, Vol. 21, No. 24, 2001, pp. 9896-9903.

[2] K. Okawa, T. Ichinohe and Y. Kaneko, "Anxiety May Enhance Pain during Dental Treatment," The Bulletin of Tokyo Dental College, Vol. 46, No. 3, 2005, pp. 51-58. doi:10.2209/tdcpublication.46.51

[3] M. Funahashi, Y.-F. He, T. Sugimoto and R. Matsuo, "Noxious Tooth Pulp Stimulation Suppresses c-fos Expression in the Rat Hippcampal Formation,” Brain Research, Vol. 827, 1999, pp. 215-220. doi:10.1016/S0006-8993(99)01250-0

[4] M. Hasegawa, J. Hada, T. Abe, K. Honda, A. Shimizu and M. Urade, "Theophylline Attenuates Hippocampal Blood Flow Responses Induced by Tooth Pulp Stimulation in Rats," Neuroscience Research, Vol. 65, No. 2, 2009, pp. 156-159. doi:10.1016/j.neures.2009.06.009

[5] K. Caesar, N. Akgoren, C. Mathiesen and M. Lariyzen, 
"Modification of Activity-Dependent Increases in Cerebellar Blood Flow by Extracellular Potassium in Anaesthetized Rats,” Journal of Physiology, Vol. 520, No. 1, 1999, pp. 281-292.

doi:10.1111/j.1469-7793.1999.00281.x

[6] U. Lindauer, D. Megow, H. Matsuda and U. Dirnagl, "Nitric Oxide: A Modulator, but Not a Mediator, of Neurovascular Coupling in Rat Somatosensory Cortex," American Journal of Physiology, Vol. 277, No. 2, 1999, pp. H799-H811.

[7] M. Zonta, M. C. Angulo, S. Gobbo, B. Rosengarten, K.-A. Hossman, T. Pozzan, and G. Carmignoto, "Neuron-to-Astrocyte Signaling Is Central to the Dynamic Control of Brain Microcirculation,” Nature Neuroscience, Vol. 6, No. 1, 2003, pp. 43-50. doi:10.1038/nn980

[8] H. Suyama, M. Kawamoto, S. Gaus and O. Yuge, "Effect of Etodolac, a COX-2 Inhibitor, on Neuropathic Pain in a Rat Model,” Brain Research, Vol. 1010, No. 1-2, 2004, pp. 144-150. doi:10.1016/j.brainres.2004.03.014

[9] K. Niwa, E. Araki, S. G. Morham, M. E. Ross and C. Iadecola, "Cyclooxygenase-2 Contributes to Functional
Hyperemia in Whisker-Barrel Cortex," Journal of Neuroscience, Vol. 20, No. 2, 2000, pp. 763-770.

[10] R. N. Dubois, S. B. Abramson, L. Grottrd, R. A. Gupta, L. S. Simon, L. B. A. van de Putte and P. E. Lipsky, "Cyclooxygenase in Biology and Disease," The FASEB Journal, Vol. 12, No. 12, 1998, pp. 1063-1073.

[11] W. E. Kaufmann, P. F. Worley, J. Pegg, M. Bremer, and P. Isakson, "COX-2, a Synaptically Induced Enzyme, Is Expressed by Excitatory Neurons at Postsynaptic Sites in Rat Cerebral Cortex," Proceeding of the National Academy of Sciences of the United States of America, Vol. 93, No. 6, 1996, pp. 2317-2321.

[12] A. J. Gelsema, D. J. Mckitrick and F. R. Calaresu, "Cardiovascular Responses to Chemical and Electrical Stimulation of Amygdala in Rats," American Journal of Physiology, Vol. 253, No. 5, 1987, pp. R712-R718.

[13] T. Seidenbecher, T. R. Laxmi, O. Stork and H.-C. Page, "Synchronization of Amygdalar and Hippocampal Theta Oscillations during Retrieval of Pavlovian Fear Memory," Science, Vol. 301, No. 5634, 2003, pp. 846-850. doi:10.1126/science.1085818 